\title{
Weak ferromagnetism and magnetoelectric coupling in bismuth ferrite
}

\author{
Claude Ederer* and Nicola A. Spaldin \\ Materials Research Laboratory and Materials Department, University of California, Santa Barbara, California 93106, USA \\ (Received 15 December 2004; published 8 February 2005)
}

\begin{abstract}
We analyze the coupling between the ferroelectric and magnetic order parameters in the magnetoelectric multiferroic $\mathrm{BiFeO}_{3}$ using density functional theory within the local spin density approximation (LSDA) and the LSDA+U method. We show that weak ferromagnetism of the Dzyaloshinskii-Moriya type occurs in this material, and we analyze the coupling between the resulting magnetization and the structural distortions. We explore the possibility of electric-field-induced magnetization reversal and show that, although it is unlikely to be realized in $\mathrm{BiFeO}_{3}$, it is not in general impossible. Finally, we outline the conditions that must be fulfilled to achieve switching of the magnetization using an electric field.
\end{abstract}

DOI: 10.1103/PhysRevB.71.060401

PACS number(s): 75.80.+q, 77.80.Fm, 81.05.Zx

There has been increasing recent interest in magnetoelectric multiferroics, ${ }^{1-5}$ which are materials that show spontaneous magnetic and electric ordering in the same phase. In addition to the fascinating physics resulting from the independent existence of two or more ferroic order parameters in one material, ${ }^{6}$ the coupling between magnetic and electric degrees of freedom gives rise to additional phenomena. The linear and quadratic magnetoelectric (ME) effects, in which a magnetization linear or quadratic in the applied field strength is induced by an electric field (or an electric polarization is induced by a magnetic field), are already well established. ${ }^{5}$ Recently, more complex coupling scenarios have been investigated. Examples are the coupling of the antiferromagnetic and ferroelectric domains in hexagonal $\mathrm{YMnO}_{3},{ }^{1}$ or the large magnetocapacitance near the ferromagnetic Curie temperature in ferroelectric $\mathrm{BiMnO}_{3}{ }^{3}$ Especially interesting are scenarios where the direction of the magnetization or electric polarization can be modified by an electric or magnetic field, respectively. Such a coupling would open up entirely new possibilities in data storage technologies, such as ferroelectric memory elements that could be read out nondestructively via the accompanying magnetization. Some progress has been made in this direction. Recently, the small $\left(0.08 \mu \mathrm{C} / \mathrm{cm}^{2}\right)$ electric polarization in perovskite $\mathrm{TbMnO}_{3}$ was rotated by $90^{\circ}$ using a magnetic field at low temperatures $(\sim 10-20 \mathrm{~K}) .{ }^{4}$ Conversely, early work on nickel-iodine boracite $^{7}$ showed that, below $\sim 60 \mathrm{~K}$, reversal of the spontaneous electric polarization rotates the magnetization by $90^{\circ}$, indicating that the axis of the magnetization, but not its sense, can be controlled by an electric field. In fact, it was believed $^{8,9}$ that electric-field-induced $180^{\circ}$ switching of the magnetization should be impossible, because a reversal of the magnetization corresponds to the operation of time inversion, whereas the electric field is invariant under this operation. In this work we show that such behavior is not generally impossible by using multiferroic bismuth ferrite, $\mathrm{BiFeO}_{3}$, as a test case to analyze the coupling between magnetism and ferroelectricity.

$\mathrm{BiFeO}_{3}$ has long been known to be, in its bulk form, an antiferromagnetic, ferroelectric multiferroic, ${ }^{10,11}$ with antiferromagnetic Néel temperature $T_{\mathrm{N}} \sim 643 \mathrm{~K}$, and ferroelectric Curie temperature $T_{\mathrm{C}} \sim 1103 \mathrm{~K}$. It has a rhombohedrally dis- torted perovskite structure with space group $R 3 c$ (see Fig. 1). ${ }^{12,13}$ The Fe magnetic moments are coupled ferromagnetically within the pseudocubic (111) planes and antiferromagnetically between adjacent planes (so-called G-type antiferromagnetic order). If the magnetic moments are oriented perpendicular to the [111] direction, the symmetry also permits a canting of the antiferromagnetic sublattices resulting in a macroscopic magnetization, so-called weak ferromagnetism. ${ }^{14,15}$ However, superimposed on the antiferromagnetic ordering, there is a spiral spin structure in which the antiferromagnetic axis rotates through the crystal with an incommensurate long-wavelength period of $\sim 620 \AA .{ }^{16}$ This spiral spin structure, which can be suppressed by doping, ${ }^{17}$ leads to a cancellation of the macroscopic magnetization and also inhibits the observation of the linear ME effect. ${ }^{18}$ However, significant magnetizations (between $0.1 \mu_{\mathrm{B}}$ and $1 \mu_{\mathrm{B}}$ per $\mathrm{Fe}),{ }^{2,19}$ as well as a strong $\mathrm{ME}$ coupling, ${ }^{2}$ have been reported recently in high-quality epitaxial thin films. This suggests that the spiral spin structure is also suppressed in thin films, perhaps due to epitaxial constraints or enhanced anisotropy. Since these epitaxial films also show large electric polarization $\left(\sim 50-60 \mu \mathrm{C} / \mathrm{cm}^{2}\right)$, they are promising candidate materials for $\mathrm{ME}$ device applications. In this work we calculate the magnetic properties of $\mathrm{BiFeO}_{3}$ and analyze the coupling between magnetism and ferroelectricity in this material.

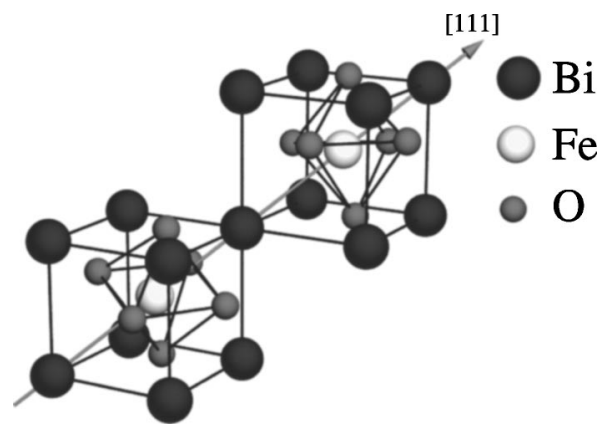

FIG. 1. Schematic view of the $R 3 c$ structure built up from two cubic perovskite unit cells. The cations are displaced along the [111] direction relative to the anions, and the oxygen octahedra rotate with alternating sense around the [111] axis. 
Our approach is based on density functional theory (DFT); see, e.g., Ref. 20, and we use two different implementations to cross check our results: the projectoraugmented plane-wave (PAW) method ${ }^{21}$ implemented in the VIENNA AB-INITIO SIMULATION PACKAGE (VASP), ${ }^{22,23}$ and the linear muffin-tin orbital method in the atomic sphere approximation (LMTO-ASA), ${ }^{24}$ extended for the treatment of noncollinear spin configurations and spin-orbit coupling. ${ }^{25,26}$ These two features, which are also implemented in the VASP code, are often omitted in DFT calculations, but are essential for our investigations. Except where explicitly stated, we use the crystal structure obtained by optimizing the atomic positions within the experimentally observed $R 3 c$ symmetry (see Fig. 1). ${ }^{12,13}$ Our calculated structural parameters are identical (within the usual numerical accuracy) to those given in Ref. 2 and agree well with the experimentally observed structure. We suppress the spiral spin structure in our calculations, consistent with the observation of a net magnetic moment in thin films. ${ }^{2,19}$ However, we emphasize that the local magnetic properties should not be strongly affected by the longwavelength spiral observed in bulk samples. ${ }^{16}$ In addition, although we use the ideal bulk symmetry throughout this paper, we have verified that our conclusions are qualitatively unchanged by the small structural changes reported in thin films. We also use two different treatments of the exchangecorrelation functional: the standard local spin-density approximation (LSDA; see Ref. 20) and the LSDA+U method. ${ }^{27}$ The LSDA+U method introduces two parameters to the treatment of the Fe $d$ states: the Hubbard parameter, $U$, and the exchange interaction, $J$. We use a value of $J=1 \mathrm{eV}$ and treat $U$ as a free parameter, varying it from 1 to $7 \mathrm{eV}$, while keeping the structure fixed to that calculated within the LSDA. For the PAW calculations we use potentials with 15 valence electrons for $\mathrm{Bi}\left(5 d^{10} 6 s^{2} 6 p^{3}\right), 14$ for $\mathrm{Fe}\left(3 p^{6} 3 d^{6} 4 s^{2}\right)$, and 6 for $\mathrm{O}\left(2 s^{2} 2 p^{4}\right)$, and a plane-wave cutoff of $400 \mathrm{eV}$. For the Brillouin-zone integrations we use a $5 \times 5 \times 5$ Monkhorst-Pack k-point mesh ${ }^{28}$ and the tetrahedron method with Blöchl correction ${ }^{29}$ (both PAW and LMTO). These values result in good convergence for all quantities under consideration.

We first investigate the occurrence and origin of weak ferromagnetism in $\mathrm{BiFeO}_{3}$. Weak ferromagnetism is intimately connected with the symmetry of the system. ${ }^{14}$ In $\mathrm{BiFeO}_{3}$ it can only occur if the sublattice magnetizations are oriented in the (111) plane so that the symmetry is reduced to the magnetic space group $B b$ or $B b^{\prime}$, which (apart from the primitive translations) contains only one glide plane. In this case a canting of the magnetic sublattices does not lead to a further reduction in symmetry and weak ferromagnetism can occur. We note that since $B b$ is a monoclinic group, even a possible monoclinic distortion of the crystal structure in the thin films would only lead to small quantitative changes in the following analysis. To determine the exact magnetic space group of $\mathrm{BiFeO}_{3}$, we calculate the preferred orientation of the sublattice magnetizations by calculating the energy difference between the arrangements with (i) magnetic moments aligned parallel or antiparallel to the [111] direction and (ii) magnetic moments oriented within the (111) plane. Both our methods result in a LSDA energy difference of about $2 \mathrm{meV}$, with a preferred orientation of the magnetic
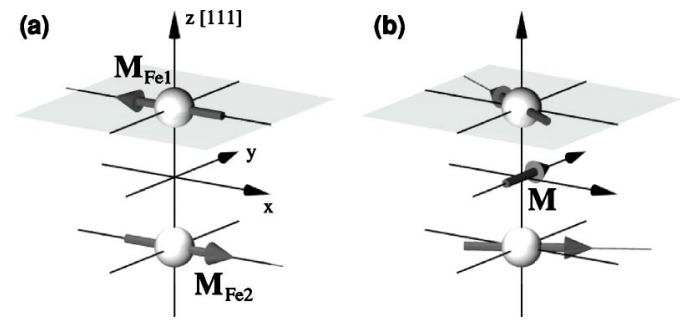

FIG. 2. (a) Starting configuration of our calculation. The magnetic moments, $\mathbf{M}_{\mathrm{Fe} 1}$ and $\mathbf{M}_{\mathrm{Fe} 2}$, of the two iron atoms in the unit cell are oriented antiferromagnetically and collinearly in the (111) plane, allowing weak ferromagnetism by symmetry. (b) Calculated magnetic structure including the spin-orbit interaction: The two iron magnetic moments rotate in the (111) plane so that there is a resulting spontaneous magnetization, $\mathbf{M}$.

moments within the (111) plane. This arrangement is compatible with the existence of weak ferromagnetism. Within the (111) plane, orientations of the sublattice magnetizations parallel or perpendicular to the glide plane are energetically equivalent. The anisotropy energy is reduced to more realistic values within the $\mathrm{LSDA}+\mathrm{U}$ method $(0.5 \mathrm{meV}$ for $U$ $=3 \mathrm{eV}, 0.2 \mathrm{meV}$ for $U=5 \mathrm{eV}$ ), but the easy magnetization orientation is unchanged.

The anisotropy calculations show only that weak ferromagnetism is symmetry allowed, not that it will actually occur. Therefore, we next calculate the magnitude of the effect. We initiate our calculations to a homogeneous and collinear spin arrangement with the magnetic moments oriented in the (111) plane [along either the $x$ - or $y$ axis in our coordinate system; see Fig. 2(a)], then let the magnetic moments relax freely within the self-consistency cycle. The magnetic moments then cant away from the collinear direction (while remaining in the (111) plane) by an angle of about $1^{\circ}$ [LSDA, Fig. 2(b)]. This leads to a small but measurable magnetization of approximately $0.1 \mu_{\mathrm{B}}$ per unit cell. LSDA $+\mathrm{U}$ calculations give the same qualitative results but with slightly smaller magnetizations (reduced by $10 \%-20 \%$ depending on the value of $U$ ). This value is smaller than that reported in Ref. [2] but agrees well with more recent measurements. ${ }^{19}$

According to Dzyaloshinskii and Moriya (DM), ${ }^{14,15}$ the canting of the magnetic sublattices is caused by an antisymmetric spin coupling, the so-called DM interaction, which is due to the combined action of exchange interaction and spinorbit coupling. Indeed, if we neglect the spin-orbit interaction in our calculations the magnetic moments remain collinear and there is no macroscopic magnetization. The DM interaction has the form

$$
E_{\mathrm{DM}}=-\frac{1}{2} \mathbf{D} \cdot\left(\mathbf{M}_{\mathrm{Fe} 1} \times \mathbf{M}_{\mathrm{Fe} 2}\right)=-\mathbf{D} \cdot(\mathbf{L} \times \mathbf{M}),
$$

where $\mathbf{D}$ is a coupling vector analogous to the Heisenberg exchange constant $J$ in the usual symmetric exchange interaction. The antiferromagnetic vector $\mathbf{L}=\mathbf{M}_{\mathrm{Fe} 1}-\mathbf{M}_{\mathrm{Fe} 2}$ is defined as the difference of the two sublattice magnetizations, and $\mathbf{M}=\mathbf{M}_{\mathrm{Fe} 1}+\mathbf{M}_{\mathrm{Fe} 2}$ is the resulting magnetization. From the form of $E_{\mathrm{DM}}$ it is clear that, for constant $\mathbf{D}$ and fixed orientation of $\mathbf{L}$, the canting of the magnetic sublattices always 
occurs such that $\mathbf{D}$ (required by symmetry to be oriented along the [111] axis), $\mathbf{L}$, and $\mathbf{M}$ build up a right-handed system. Indeed, if we start our calculation with a spin configuration in which the magnetic moments are canted in the wrong direction (so that $\mathbf{D}, \mathbf{L}$, and $\mathbf{M}$ make up a left-handed system) the moments relax back into the right-handed configuration during the iteration process. Therefore, for a particular orientation of $\mathbf{D}$ and $\mathbf{L}$, only one canting direction lowers the energy relative to the collinear state.

Next, we analyze the relationship between the weak ferromagnetism and the structural distortions in $\mathrm{BiFeO}_{3}$. As already mentioned, weak ferromagnetism depends crucially on the symmetry of the system, which in turn is determined by the structural distortions. The ferroelectric $R 3 c$ structure of $\mathrm{BiFeO}_{3}$ is reached from the ideal cubic perovskite structure by freezing in two unstable normal modes: (i) the polar displacements of all the anion and cation sublattices relative to each other, which lead to the spontaneous electric polarization, and (ii) an antiferrodistortive rotation of the oxygen octahedra around the [111] direction with alternating sense of rotation along the [111] axis (see Fig. 1). In terms of symmetry groups, the polar displacements alone would reduce the symmetry of the ideal perovskite structure $(P m \overline{3} m)$ to the rhombohedral space group $R 3 m$, whereas the rotation of the oxygen octahedra alone would lead to space group $R \overline{3} c$. The incorporation of both distortions gives the actual space group of $\mathrm{BiFeO}_{3}, R 3 c$. Weak ferromagnetism is only allowed by symmetry in the space groups $R \overline{3} c$ and $R 3 c$, suggesting that it is related to the oxygen rotations rather than to the polar displacements along [111]. We have verified this by performing calculations for structures containing only one of the two distortions (while keeping the lattice vectors fixed to those of the $R 3 c$ structure). These calculations confirm that the polar $R 3 m$ structure does not show weak ferromagnetism, whereas the nonferroelectric $R \overline{3} c$ does.

To fully understand the coupling between the structural distortions and the magnetization, we next invert the sense of rotation of the oxygen octahedra while keeping the polar distortions fixed. Again, we start from the magnetic configuration shown in Fig. 2(a), i.e., with the same orientation of $\mathbf{L}$ as in the previous calculations. In this case the magnetization direction is reversed from that of the original structure. Conversely, if we invert the polar distortion while keeping the rotational sense of the oxygen octahedra fixed, the magnetization direction is unchanged. This clearly shows that the direction of the DM vector $\mathbf{D}$ is determined by the sense of rotation of the oxygen octahedra surrounding the corresponding magnetic ions, rather than by the direction of the polarization as suggested in Ref. 30 .

Next, we investigate the switching possibilities of the system from one stable orientation state to another by the application of an electric field. A general scheme for the derivation of all possible orientation states in ferroic materials was developed by Aizu, ${ }^{31}$ who showed that the stable states can be constructed by applying all symmetry elements that are lost during the ferroic phase transition to an arbitrary orientation state of the ferroic phase. A complication arises in the case of $\mathrm{BiFeO}_{3}$ because the symmetry change between the nonferroic prototype phase (nonmagnetic $P m \overline{3} m$ ) and the fi-
TABLE I. All possible orientation states with parallel or antiparallel direction of $\mathbf{M}$. The first row gives the lost symmetry element that maps state 1 onto state $i . \overline{1}$ stands for space inversion, a prime indicates time inversion, $m$ is the mirror plane parallel to the [111] direction, and 2 is a twofold axis perpendicular to $m$. The states 5-8 are the antiphase domains not included in Aizu's scheme (see the text). The directions of $\mathbf{P}, \mathbf{D}, \mathbf{M}$, and $\mathbf{L}$ projected on the (111) plane are indicated by arrows. $\odot(\otimes)$ indicates orientation along the positive (negative) [111] axis.

\begin{tabular}{ccccccccc}
\hline \hline & 1 & $\overline{1}$ & $1^{\prime}$ & $\overline{1}^{\prime}$ & $m$ & $2(=\overline{1} m)$ & $m^{\prime}$ & $2^{\prime}$ \\
$i$ & 1 & 2 & 3 & 4 & 5 & 6 & 7 & 8 \\
\hline $\mathbf{P}$ & $\odot$ & $\otimes$ & $\odot$ & $\otimes$ & $\odot$ & $\otimes$ & $\odot$ & $\otimes$ \\
$\mathbf{D}$ & $\odot$ & $\otimes$ & $\odot$ & $\otimes$ & $\otimes$ & $\odot$ & $\otimes$ & $\odot$ \\
$\mathbf{M}$ & $\rightarrow$ & $\rightarrow$ & $\leftarrow$ & $\leftarrow$ & $\leftarrow$ & $\leftarrow$ & $\rightarrow$ & $\rightarrow$ \\
$\mathbf{L}$ & $\downarrow$ & $\uparrow$ & $\uparrow$ & $\downarrow$ & $\downarrow$ & $\uparrow$ & $\uparrow$ & $\downarrow$ \\
\hline \hline
\end{tabular}

nal multiferroic phase $(R 3 c)$ is not purely ferroic in nature. The unit cell doubling, caused by the oxygen rotations, transforms the mirror plane parallel to the [111] direction into a glide plane, and leads to the formation of antiphase domains which are not described by Aizu's scheme. To include the antiphase domains one has to consider the full space group symmetry instead of only the point group symmetry; for $\mathrm{BiFeO}_{3}$ this analysis leads to a total of 96 orientation states, which can be divided into four groups with distinct rhombohedral (polar) axes (each group containing 24 states). For each polar axis, the corresponding easy magnetization axis is threefold degenerate; this leads to eight different orientation states with parallel or antiparallel orientation of magnetization and polarization, respectively, which are relevant for the $180^{\circ}$ switching of the magnetization. These states are listed in Table I indicated by the different orientations of $\mathbf{M}, \mathbf{L}, \mathbf{D}$, and $\mathbf{P}$, where $\mathbf{P}$ is the electric polarization and the "sense" of the oxygen rotations is indicated by $\mathbf{D}$.

The application of a polarization-reversing electric field could in principle drive the system from initial state 1 into any of the degenerate states $2,4,6$, or 8 . Of course, in reality the system will prefer to change into the state separated from the initial state by the lowest energy barrier. A reversal of $\mathbf{L}$ is unlikely since it involves a $\sim 180^{\circ}$ rotation of the sublattice magnetizations, which is hindered by the magnetic anisotropy; in contrast, the reversal of $\mathbf{M}$ requires only a small reorientation of the magnetic moments. This reduces the probable outcomes to either state 4 (in which both $\mathbf{D}$ and $\mathbf{M}$ reverse) or state 8 (in which $\mathbf{D}$ and $\mathbf{M}$ are unchanged). Of these, state 8 , in which the magnetic ordering is unchanged compared to state 1 , is the most likely, since the reversal of the oxygen rotations is energetically costly, and is not required by the reversal of $\mathbf{P}$. It is clear, however, that the earlier argument ${ }^{8,9}$ that the mutual invariance under timeand space inversion of electric field and magnetization inhibits the possibility of electric-field-induced magnetization reversal, does not hold. While it is certainly correct that it is not possible to drive the system from state 1 to its timeconjugate state $1^{\prime}$ using an electric field, the state corresponding to $\overline{1}^{\prime}$ is also present in most ferroelectric ferromag- 
nets. If the energy barrier for the transition to $\overline{1}^{\prime}$ or to any other orientation state with reversed $\mathbf{M}$ and $\mathbf{P}$ is lower than the energy barrier to all other degenerate orientation states, then the magnetization will be invertible by an electric field.

From the above discussion we can extract three conditions that must be fulfilled to achieve electric-field-induced magnetization reversal in such a rhombohedrally distorted multiferroic perovskite: (i) the rotational and polar distortions must be coupled; (ii) the degeneracy between states 1, 2, 3, 4 and 5, 6, 7, 8 must be lifted, i.e., parallel and antiparallel orientations of $\mathbf{D}$ and $\mathbf{P}$ must be inequivalent; and (iii) there must be only one easy magnetization axis in the (111) plane. The latter condition can easily be achieved by straining the material in an appropriate way.
In summary, we have shown that $\mathrm{BiFeO}_{3}$ exhibits weak ferromagnetism of the DM type if the spiral spin structure is suppressed. We have also shown that the DM vector is determined by the rotations of the oxygen octahedra rather than by the ferroelectric polarization. Finally, we have discussed the possible magnetoelectric switching scenarios in $\mathrm{BiFeO}_{3}$, and formulated conditions that must be met to realize electric-field-induced magnetization reversal.

The authors thank H. Schmid and D. Vanderbilt for valuable discussions. This work was supported by the MRSEC program of the National Science Foundation under Award No. DMR00-80034.
*Electronic address: ederer@mrl.ucsb.edu

${ }^{1}$ M. Fiebig, T. Lottermoser, D. Fröhlich, A. V. Goltsev, and R. V. Pisarev, Nature (London) 419, 818 (2002).

${ }^{2}$ J. Wang et al., Science 299, 1719 (2003).

${ }^{3}$ T. Kimura, S. Kawamoto, I. Yamada, M. Azuma, M. Takano, and Y. Tokura, Phys. Rev. B 67, 180401(R) (2003).

${ }^{4}$ T. Kimura, T. Goto, H. Shintani, K. Ishizaka, T. Arima, and Y. Tokura, Nature (London) 426, 55 (2003).

${ }^{5}$ Proceedings of the 5th International Workshop on Magnetoelectric Interaction Phenomena in Crystals, edited by M. Fiebig, V. Eremenko, and I. E. Chupis (Kluwer, Dordrecht, 2004).

${ }^{6}$ N. A. Hill, Annu. Rev. Mater. Res. 32, 1 (2002).

${ }^{7}$ E. Ascher, H. Rieder, H. Schmid, and H. Stössel, J. Appl. Phys. 37, 1404 (1966).

${ }^{8}$ H. Schmid, Ferroelectrics 221, 9 (1999).

${ }^{9} \mathrm{H}$. Schmid, in Introduction to Complex Mediums for Optics and Electromagnetics, edited by W. S. Weiglhoger and A. Lakhtakia (SPIE, Bellingham, WA, 2003).

${ }^{10}$ S. V. Kiselev, R. P. Ozerov, and G. S. Zhdanov, Sov. Phys. Dokl. 7, 742 (1963).

${ }^{11}$ J. R. Teague, R. Gerson, and W. J. James, Solid State Commun. 8, 1073 (1970).

${ }^{12}$ C. Michel, J.-M. Moreau, G. D. Achenbach, R. Gerson, and W. J. James, Solid State Commun. 7, 701 (1969).

${ }^{13}$ F. Kubel and H. Schmid, Acta Crystallogr., Sect. B: Struct. Sci. 46, 698 (1990).
${ }^{14}$ I. E. Dzyaloshinskii, Sov. Phys. JETP 5, 1259 (1957).

${ }^{15}$ T. Moriya, Phys. Rev. 120, 91 (1960).

${ }^{16}$ I. Sosnowska, T. Peterlin-Neumaier, and E. Streichele, J. Phys. C 15, 4835 (1982).

${ }^{17}$ I. Sosnowska, W. Schäfer, W. Kockelmann, K. H. Andersen, and I. O. Troyanchuk, Appl. Phys. A: Mater. Sci. Process. 74, S1040 (2002).

${ }^{18}$ Y. F. Popov, A. K. Zvezdin, G. P. Vorob'ev, A. M. Kadomtseva, V. A. Murashev, and D. N. Rakov, JETP Lett. 57, 69 (1993).

${ }^{19} \mathrm{~W}$. Eerenstein and N. Mathur (unpublished).

${ }^{20}$ R. O. Jones and O. Gunnarsson, Rev. Mod. Phys. 61, 689 (1989).

${ }^{21}$ P. E. Blöchl, Phys. Rev. B 50, 17953 (1994).

${ }^{22}$ G. Kresse and J. Furthmüller, Phys. Rev. B 54, 11169 (1996).

${ }^{23}$ G. Kresse and D. Joubert, Phys. Rev. B 59, 1758 (1999).

${ }^{24}$ O. K. Andersen, Phys. Rev. B 12, 3060 (1975).

${ }^{25}$ O. Grotheer, C. Ederer, and M. Fähnle, Phys. Rev. B 63, 100401(R) (2001).

${ }^{26}$ C. Ederer, M. Komelj, M. Fähnle, and G. Schütz, Phys. Rev. B 66, 094413 (2002).

${ }^{27}$ V. I. Anisimov, F. Aryasetiawan, and A. I. Liechtenstein, J. Phys.: Condens. Matter 9, 767 (1997).

${ }^{28}$ H. J. Monkhorst and J. D. Pack, Phys. Rev. B 13, 5188 (1976).

${ }^{29}$ P. E. Blöchl, O. Jepsen, and O. K. Andersen, Phys. Rev. B 49, 16223 (1994).

${ }^{30}$ D. L. Fox and J. F. Scott, J. Phys. C 10, L329 (1977).

${ }^{31}$ K. Aizu, Phys. Rev. B 2, 754 (1970). 